\title{
NEUROSCIENCE
}

\section{Optogenetics turns up the heat}

Illuminating the brain for optogenetic experiments can have undesirable effects on neural activity.

O ptogenetics and fluorescence imaging have become workhorses in neuroscience. However, these technologies require illumination of brain tissue with sufficient light intensities to manipulate or visualize neuronal activity, which goes hand in hand with increased temperature in the tissue.

Anatol Kreitzer from the Gladstone Institutes in San Francisco and his colleagues have assessed how these temperature changes affect neuronal activity in different brain regions and neuronal cell types. They recorded from medium spiny neurons in the striatum, granule cells in the dentate gyrus, fastspiking interneurons in the cortex, and pyramidal cells in the hippocampus and cortex, while illuminating these brain regions with intensities typically used in optogenetic experiments.
Whereas pyramidal cells were resilient under illumination, the other cell types reduced their firing rates in response to the increased temperature caused by the illumination. These effects arose in the absence of expression of optogenetic actuators. The extent of the firing rate reduction correlated with the extent of the temperature increase. Importantly, these changes in firing rate can have behavioral effects. For instance, illumination of the dorsal striatum in one side of the brain caused the mice to bias their turning behavior toward the same side, as expected for a suppression of this brain region. However, if inhibitory interneurons are predominantly affected by heat, the activity of a targeted brain area can also be increased on the whole.

Kreitzer and his team determined that the thermosensitivity of neurons is mediated by an inward-rectifying potassium channel, which is consistent with their electrophysiological characterization of the temperature effect.

These studies underscore the importance of being mindful of heat generation during illumination of the brain. But published theoretical models can help predict the expected temperature increase depending on illumination power, wavelength, duty cycle and other parameters, and optimize illumination protocols.

Nina Vogt

Published online: 30 July 2019

https://doi.org/10.1038/s41592-019-0528-2

\section{Research papers}

Owen, S. F. et al. Thermal constraints on in vivo optogenetic manipulations. Nat. Neurosci. 22, 1061-1065 (2019).

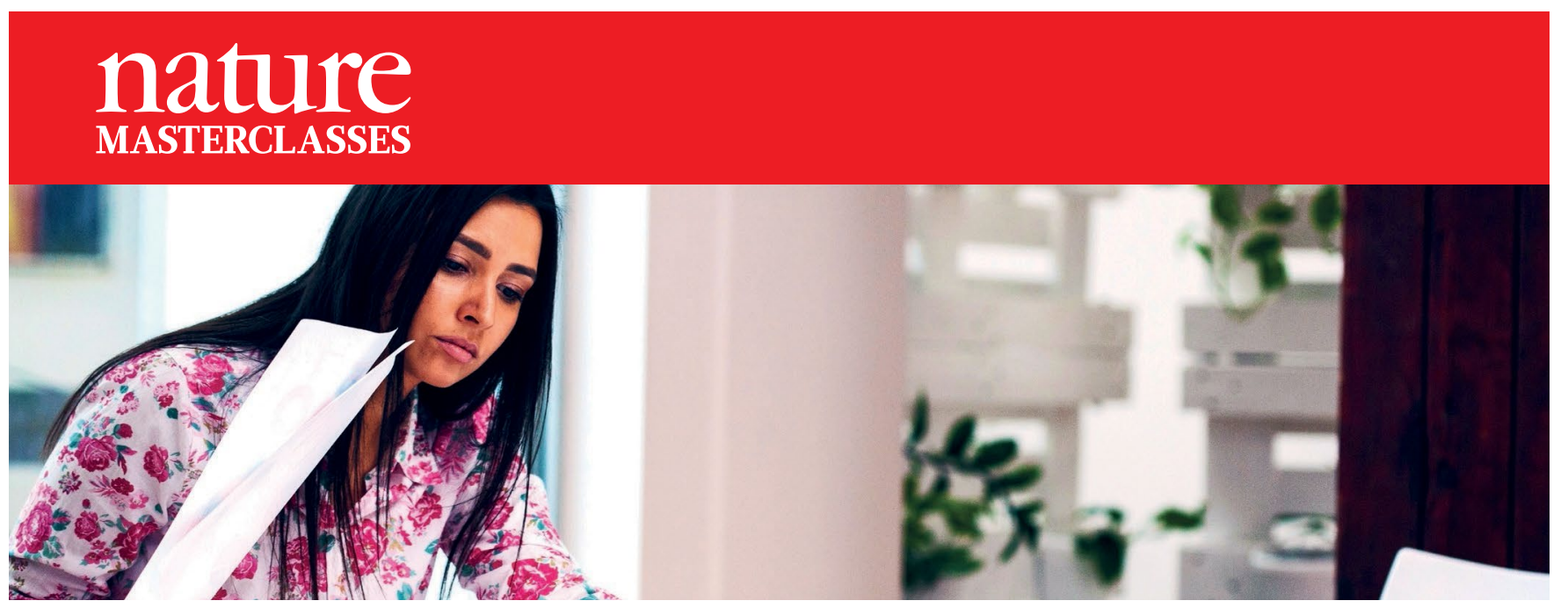

\section{Free online course for peer reviewers}

For researchers new to peer review or wishing to develop their skills

\section{Register for free access at masterclasses.nature.com}

\section{Taught by Nature Research editors • 3-4 hours' learning • Free completion certificate}

\section{W masterclasses.nature.com \\ in Follow us on Linkedln \\ f Skills and Careers Forum for Researchers}

